\title{
Assistance au suicide: quel rôle pour le médecin?
}

\section{Hans Stalder}

Prof. Dr méd., membre de la rédaction

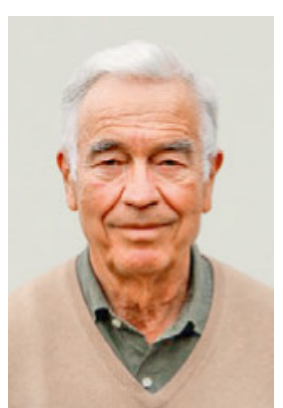

Scénario 1: J'ai un ami qui veut se suicider et me demande de l'aider. "Peux-tu me passer ton pistolet d'ordonnance?» me demande-t-il et il ajoute que je n'aurai aucun ennui, car la loi ne poursuit l'aide au suicide que s'il y a un motif égoïste [1]. Comme médecin j'aurais un moyen beaucoup moins violent, mais je n'ose pas lui en parler.

Scénario 2: Mon patient de 80 ans est fatigué de vivre. Sa femme étant décédée, ses enfants quasi absents, il refuse d'aller dans un EMS. Je lui réponds que selon la déontologie médicale actuelle, je ne puis l'aider que si son décès est proche, mais que selon les nouvelles directives [2], je devrais m'assurer que ses souffrances sont insupportables, en cas de doute appeler un spécialiste pour évaluer sa capacité de discernement et consulter une tierce personne pour confirmer que son désir de mourir est mûrement réfléchi. Mon patient me répond: «Pourquoi toutes ces conditions? Vous me connaissez de longue date et j'ai pleine confiance en vous.»

Scénario 3: Cette patiente souffre d'un cancer avec métastases et des douleurs intenses. Elle me demande de l'aider à mourir. Je lui réponds qu'on peut traiter les douleurs d'une manière efficace. Je pourrais augmenter les antidouleurs et les psychotropes même si elle en meurt. Elle me répond: "Je préfère mourir avec votre aide, réveillée et en présence de mes proches.»

Ces vignettes servent à discuter trois points qui à mon avis n'ont pas ou pas suffisamment été traités dans les nouvelles directives de l'ASSM «Attitude face à la fin de vie et à la mort» [2]. Certes, elles constituent un pas en avant comparées aux anciennes, qui ne permettaient l'assistance au suicide qu'en cas de mort imminente.

Le scénario 1 montre qu'il y a divergence entre la déontologie médicale concernant l'assistance au suicide et la loi, qui ne poursuit qu'en cas de motif égoïste. La prescription de pentobarbital est sans doute la façon la plus humaine d'aider à mourir, pas seulement pour la personne, mais aussi pour les proches. Seuls les médecins peuvent le prescrire et le procurer. L'aide au suicide est donc par défaut bel et bien devenue une «nouvelle tâche» et ne relève pas "de la compétence d'autres acteurs sociaux" [3]. Nous sommes ici dans une situation similaire à celle qui prévalait il y a quelques décennies pour l'avortement: bien plus humain fait par un gynécologue plutôt qu'avec des moyens artisanaux. Il est évident que, comme pour l'avortement, l'assistance au suicide ne doit être pratiquée qu'après une discussion approfondie et empathique.

Le scénario 2 concerne l'autonomie du patient. Qui décide que les souffrances sont insupportables? N'est-ce pas une attitude patriarcale d'en faire une décision médicale visant plutôt à rassurer le médecin qu'à aider le patient? De plus ces directives risquent de prolonger la souffrance inutilement, surtout en cas d'issue fatale proche, car elles exigent même pour le médecin de famille connaissant bien son patient, que la capacité de discernement soit évaluée en cas de doute par un spécialiste et que le désir de mort soit durable et confirmé après des entretiens répétés et par une tierce personne. Le scénario 3 montre comment nous évitons avec hypocrisie d'appeler euthanasie certains de nos actes. Extuber un patient en cas d'issue fatale ou appliquer une «sédation continue jusqu'à la mort» [2] dans une situation désespérée-des actes que nous avons tous pratiqués lors de nos stages à l'hôpital - causent activement la mort. Que faire si le patient désire mourir en pleine conscience entouré par ses proches plutôt que dans un coma dû au surdosage médicamenteux? Pour un changement de la loi sur l'euthanasie [1] il y a des pour et des contre [4], mais nous aurions aimé que ce sujet sensible soit approfondi d'abord par l'éthique avant d'être discuté par des parlementaires (ce qui va certainement arriver).

Les progrès médicaux dans la prolongation et la maintenance de la vie ainsi que les changements sociétaux ne permettant plus une attitude patriarcale posent des défis nouveaux aux médecins. Le succès d'Exit et l'attrait de Dignitas doivent nous faire réfléchir si nos règles déontologiques datant de l'aire hippocratique sont encore adaptées. D'autres pays l'ont fait: pourquoi pas la Suisse? Nous aurions aimé que la Commission d'éthique de l'ASSM aille un peu plus loin et aborde ces points délicats.

\section{Références}

1 Voir Code pénal suisse, Art. 114 et 115.

2 Attitude face à la fin de vie et à la mort. Académie Suisse des Sciences médicales. https://www.assm.ch/fr/Ethique/Ethiqueen-fin-de-vie/Directives-Fin-de-vie.html

3 Kind C. Directives révisées «Attitude face à la fin de vie et la mort». Bull Méd Suisses. 2017;98:49-50.

4 Kolatte E, Bonvallat F, Deslarzes B, et al. Euthanasie: réflexion et essai de clarification. Cahier d'uni3 12. Genève 2013. https://www. unige.ch/uni3/qui-sommes-nous/commission/publications/ 